\title{
Die Rekrutierung neuer Mitarbeiter aus Organisationsperspektive: eine komplementäre Sicht auf soziale Netzwerke im Arbeitsmarkt ${ }^{1}$
}

\section{Von Ulf Liebe und Karsten Wegerich}

Zusammenfassung: Eine Vielzahl von Studien zeigt, dass soziale Netzwerke/Kontakte Informationsprobleme im Arbeitsmarkt überwinden und für Jobsuchende von Vorteil sind. Jedoch liegen zur komplementären Bedeutung sozialer Netzwerke aus der Organisationsperspektive (Belegschaftsempfehlungen) nur wenige Befunde vor. Wir geben in einem ersten Schritt einen Überblick über den Forschungsstand, wobei Studien zum Rekrutierungsverhalten einzelner Organisationen im Mittelpunkt stehen. Solche Bewerberpoolstudien haben den Vorteil, dass Informationen über relevante Organisationsmerkmale und -anforderungen sowie alle erfolgreichen und nicht erfolgreichen Bewerbungen auf jeder Stufe im Rekrutierungsprozess verfügbar sind. In einem zweiten Schritt stellen wir eine empirische Studie auf Basis des gesamten Bewerberpools (Februar bis April 2007, $\mathrm{N}=477$ ) eines deutschen Unternehmens aus der Textil-Branche (mit Online-Vertrieb) vor. Multivariate Analysen zeigen, dass sich bei Kontrolle von Humankapital Belegschaftsempfehlungen auf den einzelnen Rekrutierungsstufen auszahlen (,,better match“ hinsichtlich Weiterleitung der Bewerbung, Jobinterview und Jobangebot). Insgesamt wird auch das spezifische Analysepotenzial von Bewerberpoolstudien, z.B. eine Verortung von (ungewollter) Diskriminierung, sichtbar.

\section{Einführung}

Es gibt Unternehmer/-innen, die bevorzugt Personen einstellen, die von Mitarbeiter/-innen im eigenen Unternehmen empfohlen werden oder mit Mitarbeiter/-innen im eigenen Unternehmen verwandt sind. ${ }^{2}$ Ein Grund hierfür dürfte sein, dass sich die Unternehmer einen geringeren Suchaufwand und mehr Klarheit über die Qualität ihrer Mitarbeiter versprechen. Für manche Arbeitgeber (auch in öffentlichen Organisationen) mag es nahe liegen, von positiven Eigenschaften des referierenden Mitarbeiters auf die Eigenschaften des empfohlenen Bewerbers zu schließen. Dass soziale Kontakte bzw. soziales Kapital auf dem Arbeitsmarkt eine Rolle spielen, wird seit langem immer wieder behauptet, zu erklären versucht und kritisch diskutiert. Während man auch dem Alltagsverständnis nach annehmen würde, dass „Vitamin B“ bei der Stellensuche und Stellenbesetzung erfolgsfördernd ist (z.B. bei der Stellenvergabe, Gehalt usw.), sprechen die empirischen Befunde keine klare Sprache (vgl. Granovetter [1974] 1995: 139ff sowie Voss 2007 für Überblicksarbeiten).

Prinzipiell können Untersuchungsansätze zur Bedeutung von sozialen Kontakten auf dem Arbeitsmarkt danach unterschieden werden, ob sie die Jobsuchenden- oder die Organisationsperspektive einnehmen. Die meisten Studien sind zweifelsfrei in der ersten Gruppe anzutreffen. Dabei wird überwiegend untersucht, auf welche Art und Weise Personen ihre derzeitige Stelle oder auch frühere Stellen gefunden haben (z.B. Granovetter [1974] 1995; Preisendörfer / Voss 1988; Wegener 1991; Jann 2003; Mouw 2003; Franzen / Hangartner 2005, 2006). Neben der Bedeutung der verwendeten Bewerbungskanäle fragen diese Studien mitunter auch, inwiefern sich die Nutzung formeller oder informeller Bewerbungskanäle z.B. auf das Einkommen oder nicht-monetäre Aspekte der Arbeitsstelle auswirkt. Jedoch haben einige Studien (z.B. Grano-

1 Die Autoren danken Thomas Voss, Peter Preisendörfer und Melanie Olczyk für wertvolle Hinweise und Anregungen zu früheren Versionen des Manuskripts.

2 Im Folgenden wird auf den Zusatz ,/-in“ bzw. „/-innen“ verzichtet. Dies dient ausschließlich der sprachlichen Vereinfachung. 
vetter [1974] 1995) den Nachteil, dass nur die erfolgreichen Bewerber (eine selektive Untersuchungspopulation) in den Blick genommen werden und keine Informationen über die Bewerbungskanäle der nicht erfolgreichen Bewerber vorliegen (vgl. Fernandez / Weinberg 1997: 884). Dieses Problem umgehen prinzipiell Untersuchungen, die auf eine Stichprobe von Stellensuchenden abstellen (z.B. Kahn / Low 1982; Holzer 1987), wobei diese wiederum auch eine selektive Population darstellen.

Rekrutierungsaktivitäten, Besonderheiten oder spezifische Anforderungen der einstellenden Organisationen nehmen insbesondere Studien aus der Organisationsperspektive in den Blick. Zum einen werden Unterschiede in der Rekrutierungsstrategie zwischen Organisationen (z.B. Firmen / Branchen) untersucht (z.B. Windolf 1986; Marsden / Campbell 1990; Holzer 1996; Marsden / Gorman 2001: 480ff für einen Überblick). Dieser Ansatz berücksichtigt u.a. auch die Umweltbedingungen (z.B. die Arbeitsmarktsituation) und Merkmale (z.B. Kapazitäten zur Informationsgewinnung) von Organisationen. In den empirischen Studien stehen jedoch lediglich erfolgreiche Rekrutierungen im Mittelpunkt, was ebenfalls auf ein Selektionsproblem hindeutet. Zum anderen wird der gesamte Bewerberpool einer einzelnen Organisation analysiert (überwiegend Firmen, z.B. Fernandez / Weinberg 1997; Petersen et al. 2000; Yakubovich / Lup 2006). Solche Studien haben den Vorteil, dass einerseits Daten zu allen erfolgreichen sowie nicht erfolgreichen Bewerbungen zur Verfügung stehen und andererseits auch die Rekrutierungsstrategie sowie spezifische Anforderungen der Organisation berücksichtigt werden können. Zudem sind in der Regel Informationen über alle Rekrutierungsstufen verfügbar. Allerdings sind die empirischen Ergebnisse nicht ohne weiteres verallgemeinerbar, da Untersuchungen auf Basis einer Organisation einen Fallstudien-Charakter haben. Mithin ergänzen sich die vorgestellten Untersuchungsansätze, die Jobsuchenden- und Organisationsperspektive, und ergeben (nur) zusammengenommen ein Gesamtbild der Bedeutung von sozialen Kontakten auf dem Arbeitsmarkt.

Der vorliegende Beitrag stellt den letztgenannten Ansatz ausführlicher vor, möchte zu weiteren Anwendungen motivieren und präsentiert eine empirische Studie auf Basis des gesamten Bewerberpools eines Unternehmens aus der Textil-Branche (Online-Zusammenstellung und -Vertrieb von Textilprodukten aus der eigenen Herstellung), das seinen Hauptsitz in den neuen Bundesländern hat. Neben Aussagen über den gesamten Bewerberpool (kein Selektionsproblem) erlaubt unsere Studie eine detaillierte Abbildung des Rekrutierungsprozesses bis zur Jobvergabe (Eingang der Bewerbung, Weitergabe an eine Fachabteilung, Einladung zum Interview, Jobangebot). Dabei können wir sowohl die Bedeutung von sozialen Kontakten als auch der Nutzung von Headhuntern zur Mitarbeiterrekrutierung empirisch nachvollziehen. Studien wie die vorliegende gibt es bisher kaum (es liegen nur fünf zentrale Studien weltweit vor und ausschließlich für die USA, mehr dazu in Abschnitt 2). Wir demonstrieren im Wesentlichen das Analysepotenzial von Bewerberpoolstudien und erweitern die empirischen Grundlagen in diesem Forschungsfeld. Damit kommen wir nicht zuletzt einer Aufforderung von Fernandez/Weinberg (1997: 886) nach, die bis heute nicht an Aktualität verloren hat: „We need studies that tap the screening process at various phases and measure the ways in which social ties might affect outcomes at each stage."

Der zweite Abschnitt gibt einen Überblick über den Forschungsstand (theoretische Mechanismen und bisherige empirische Befunde). Einen Überblick über die Datenbasis, die zentralen Variablen und deskriptive Befunde beinhaltet der dritte Abschnitt. Ergebnisse von multivariaten Analysen zu den einzelnen Rekrutierungsstufen bilden den Kern des vierten Abschnitts. Der Beitrag endet mit einigen Schlussbemerkungen im fünften Abschnitt. 


\section{Theoretischer Hintergrund und bisherige empirische Befunde}

Die Stellenbesetzung bzw. Rekrutierung von Mitarbeitern wird im vorliegenden Beitrag aus der Organisations- bzw. Arbeitgeberperspektive betrachtet. Grundsätzlich ist der MatchingProzess auf dem Arbeitsmarkt mit (erheblichen) Informationsproblemen verbunden. Zum einen müssen Arbeitgeber potenzielle Kandidaten für zu besetzende Stellen ausfindig machen. Zum anderen besteht eine Ungewissheit ex ante (vor der Jobvergabe) und ex post (nach der Jobvergabe), inwieweit potenzielle Kandidaten die Organisationsanforderungen erfüllen. Vor diesem Hintergrund können Arbeitgeber unterschiedliche Rekrutierungsstrategien verfolgen und bei der Mitarbeitersuche verschiedene Rekrutierungskanäle aktivieren. Sowohl der Suchaufwand als auch die Auswahl von Rekrutierungskanälen/-strategien ist gemeinhin von den Merkmalen (z.B. der Größe) und den Umweltbedingungen (z.B. der allgemeinen Arbeitsmarktsituation/-position) einer Organisation abhängig (u.a. Windolf 1986; Marsden / Gorman 2001; Voss 2007). Jedoch sind für alle Organisationen soziale Kontakte bzw. Belegschaftsempfehlungen ein potenzieller Rekrutierungskanal neben den gängigen formellen Rekrutierungskanälen wie Anzeigen in Zeitungen, Internetportalen usw. "Belegschaftsempfehlungen“ bedeutet hierbei, dass Mitarbeiter der suchenden Organisation Jobkandidaten empfehlen, die sie durch Arbeitskontakte oder aber durch Verwandtschafts- und Freundschaftsbeziehungen kennen. Mitunter erhalten Mitarbeiter für ihre Empfehlungen eine Prämie. ${ }^{4}$ Analog zur Perspektive der Jobsuchenden werden auch aus der Organisationsperspektive heraus Vorteile der Nutzung sozialer Kontakte bzw. soziale Mechanismen bei der Mitarbeiterrekrutierung postuliert (vgl. Voss 2007 für einen Überblick).

\subsection{Soziale Mechanismen im Rekrutierungsprozess}

Es stellt sich zunächst die Frage, welche spezifischen Vorteile aus Organisationsperspektive die Rekrutierung über soziale Kontakte, vor allem über Belegschaftsempfehlungen, erwarten lässt. Fernandez et al. (2002: 1291ff.) benennen, primär mit Blick auf Firmen/Unternehmen, fünf soziale Mechanismen, warum Belegschaftsempfehlungen Vorteile für Organisationen nach sich ziehen: ${ }^{5}$

(1) Der Bewerberpoolmechanismus zielt darauf ab, dass Belegschaftsempfehlungen den Bewerberkreis erweitern. Dieser schließt nunmehr Bewerber ein, die sich ohne eine Empfehlung bzw. unternehmensbezogene Informationen via soziale Kontakte nicht beworben hätten.

(2) Der Homophiliemechanismus beinhaltet die Überlegung, dass im Vergleich zu anderen Rekrutierungsstrategien besser qualifizierte Mitarbeiter über Empfehlungen gewonnen werden. Hintergrund ist die Annahme, dass die soziale Beziehung zwischen Bewerber und Empfehlendem durch Homophilie gekennzeichnet ist (d.h. beide gleichen sich in

3 Das Internet ist ein formeller Rekrutierungskanal, der sich von „klassischen“ Kanälen wie Anzeigen in Zeitungen unterscheidet. Zudem bietet es für Jobsucher und mitunter auch für Arbeitgeber die Möglichkeit, zusätzliche Informationen über die jeweils andere Partei zu erhalten. Allerdings muss auch hier die Qualität der Informationen entsprechend evaluiert werden. Fountain (2005) diskutiert ausführlich Aspekte der Bedeutung des Internets bei der Stellensuche und legt eine empirische Studie zu arbeitslosen Jobsuchern vor. Hierbei zeigt sich u.a., dass sich die Internetsuche lediglich auszahlt, wenn nicht zu viele andere dieses Medium ebenfalls nutzen. Insgesamt besteht zum Thema „Internet und Arbeitsmarkt" ein erheblicher Forschungsbedarf.

4 Organisationen können daher soziale Kontakte als Rekrutierungskanal aktiv fördern. Sie profitieren aber auch von sozialen Kontakten ihrer Mitarbeiter, wenn diese von sich aus Jobkandidaten empfehlen oder Personen auf offene Stellen im Unternehmen aufmerksam machen. Letzteres wäre eine passive Strategie.

5 Theoretische Vertiefungen zu den einzelnen Mechanismen, etwa suchtheoretische Argumente in Verbindung mit Überlegungen zu sozialen Netzwerken und Homophilie, finden sich u.a. in den Arbeiten von Rees (1966), Granovetter (1995), Montgomery (2001) und Mouw (2003). 
zentralen Merkmalen wie Bildung und Leistungsmotivation). Da der Empfehlende den Rekrutierungsprozess im Unternehmen bereits erfolgreich durchlaufen hat, ist es wahrscheinlich, dass dies dem Empfohlenen ebenso gelingt. Die „Bewerber-EmpfehlendenÄhnlichkeit" führt demnach dazu, dass empfohlene Bewerber besser zu den Stellenanforderungen passen und daher die Unternehmenserwartungen besser erfüllen sollten als andere Kandidaten.

(3) Der Reputationsmechanismus lässt sich damit begründen, dass Empfehlende einen Anreiz haben, besonders geeignete Jobkandidaten zu benennen, weil sonst ihre Reputation im eigenen Unternehmen leiden könnte. Anders ausgedrückt: Sobald jemand einen ungeeigneten Kandidaten empfiehlt, kann dies zu sozialer Missachtung im Unternehmen führen und womöglich den künftigen Werdegang im Unternehmen beeinträchtigen.

(4) Hinter dem Informationsmechanismus verbirgt sich die Annahme, dass Belegschaftsempfehlungen die Quantität und Qualität der Informationen erhöht, die der Jobsuchende und das Unternehmen übereinander haben. Der Empfehlende verfügt über Insiderinformationen, die für beide Matching-Parteien nützlich sind. Das Unternehmen erhält beispielsweise Informationen über die Charaktereigenschaften und persönlichen Einstellungen eines Bewerbers. Dieser wiederum erfährt beispielsweise, welche informellen Regeln und Abläufe das Geschehen im Unternehmen kennzeichnen. Diese Art der Informationen ist über formelle Rekrutierungskanäle nicht zugänglich und sollte insgesamt zu einem besseren „Match“ zwischen Jobkandidat und Unternehmen führen.

Die ersten vier Mechanismen beziehen sich auf die Rekrutierungsphase, der fünfte Mechanismus auf die Phase nach der Einstellung eines Bewerbers.

(5) Der „,Social-Enrichment"-Mechanismus lässt erwarten, dass die soziale Beziehung (z.B. eine Freundschaftsbeziehung) zwischen dem Empfehlenden und dem Empfohlenen letzterem den Einstieg und das „Einleben“ in das Unternehmen erleichtert. Fernandez et al. (2002: 1297) betonen hierbei, im Unterschied zur Idee eines besseren Match durch Belegschaftsempfehlungen, eine aktive Rolle der sozialen Beziehung: ,[...], the social enrichment account has refferers actively changing the relationship between the firm and the new hire. It is the presence of social ties that directly benefits the firm.”

Sowohl die „Better-Match“-Prozesse als auch der ,Social-Enrichment“-Prozess lassen insgesamt vermuten, dass Belegschaftsempfehlungen für Organisationen positive Effekte haben (bessere Eignung, geringere Fluktuation und höhere Produktivität als andere Jobkandidaten). Allerdings kommt bei ,social enrichment“ die Möglichkeit von unerwünschten Effekten hinzu (Fernandez et al. 2002: 1297). Die erwartete besonders gute soziale Integration von empfohlenen Bewerbern bietet das Potenzial für eine Stärkung informeller sowie unerwünschter Gruppenprozesse (z.B. Normen der Leistungsenthaltung, gegenseitiges Stillhalten beim „Blaumachen" usw.). Es besteht demnach ein gewisses Risiko für die Organisation, ob sich soziales Kapital an diesem Punkt wirklich auszahlt.

Aus theoretischer Perspektive haben Headhunter bzw. Personalvermittler (in diesem Zusammenhang bisher kaum erforscht) und soziale Kontakte als Rekrutierungskanal einige Gemeinsamkeiten. Headhunter sind in der Personalpraxis bei solchen Stellen von Bedeutung, die mit (sehr) speziellen Anforderungen verbunden sind (vgl. Holtbrügge 2005, z.B. im Topmanagement, bei Führungspositionen oder „Fachspezialisten“). Im Unterschied zu Belegschaftsempfehlungen sind Headhunter mit höheren direkten Kosten für das Unternehmen verbunden (d.h. in der Regel mit höheren Prämien für die Headhunter sowie Personalvermittler). Dennoch können auch hier Vorteile gegenüber formellen Rekrutierungskanälen angenommen werden: Headhunter erweitern den Bewerberpool, sowohl das Unternehmen als auch der Jobsuchende 
erhalten wichtige zusätzliche Informationen übereinander, und der Reputationsmechanismus sollte (mit Blick auf erwartete künftige Aufträge) wirken. ${ }^{6}$

Die von Headhuntern vermittelten Jobkandidaten sollten also mindestens genauso gut die Unternehmensanforderungen erfüllen wie über informelle Kontakte vermittelte Kandidaten, was schließlich in eine vergleichbar effiziente Bewerberauswahl und ein vergleichbar gutes Match mündet (vgl. Petersen et al. 2000 für empirische Tendenzen in diese Richtung). Sollte dies der Fall sein, dann hätten Headhunter gegenüber sozialen Kontakten den Vorteil, dass sie geeignete Bewerber praktisch auf Abruf bereitstellen können und eine größere Heterogenität des Bewerberpools erzeugen. Bei der aktiven und passiven Nutzung informeller Kanäle ist der „zahlenmäßige Eingang“" von Bewerbungen mitunter schwer zu steuern. Allerdings sind Mitarbeiter eines Unternehmens, im Gegensatz zu Headhuntern, unter Umständen besonders motiviert, nach geeigneten Jobkandidaten zu suchen, mit denen sie gut zusammenarbeiten können. Dabei ist nicht auszuschließen, dass die intrinsische Motivation, geeignete Kandidaten zu empfehlen, durch den Anreiz einer Prämie sogar verdrängt wird („,crowding out“, analog zu den Beispielen in Frey 1997). Darüber hinaus stellen sich bei Rekrutierungen über Headhunter etwaige Probleme im Zusammenhang mit dem weiter oben angesprochen „Social-Enrichment"-Mechanismus von sozialen Kontakten eher nicht (z.B. die Stärkung ungewünschter informeller Gruppenprozesse). Der ,janusköpfige Charakter“ sozialen Kapitals führt zu der über den vorliegenden Beitrag hinausgehenden Frage, unter welchen Bedingungen die Vorteile der Nutzung von Headhuntern diejenigen von Belegschaftsempfehlungen kompensieren (und vice versa). Jedoch lassen die diskutierten theoretischen Mechanismen insgesamt erwarten, dass Jobkandidaten, die über soziale Kontakte und Headhunter rekrutiert werden, auf allen Stufen eines Rekrutierungsprozesses eine höhere Wahrscheinlichkeit haben, diese Stufen erfolgreich zu absolvieren, als Jobkandidaten, die über formelle Kanäle rekrutiert werden.

\subsection{Bisherige empirische Befunde}

Die Anzahl an Studien, die sich auf den gesamten Bewerberpool einer Organisation (überwiegend Firmen / Unternehmen) beziehen und verschiedene Rekrutierungsstufen berücksichtigen, ist bislang überschaubar. Tabelle 1 gibt einen Überblick über die fünf wesentlichen empirischen Arbeiten. Die drei „Fernandez-Studien“ (Fernandez / Weinberg 1997; Fernandez et al. 2000; Fernandez / Sosa 2005) untersuchen Einstiegsjobs, Banker-Positionen oder Jobs in einem „phone center“ in einer US-amerikanischen Bank (jeweils mit einer unterschiedlichen Datenbasis). Die multivariaten Analysen umfassen zwei Rekrutierungsstufen, einerseits, ob Bewerber zu einem Jobinterview eingeladen werden und andererseits, ob Bewerber ein Jobangebot erhalten. Die Grundlage der Studie von Petersen et al. (2000) bilden Informationen zu allen Bewerbungen für Einstiegsjobs und andere Jobs in einem Hochtechnologie-Unternehmen in den USA, wobei drei Rekrutierungsstufen analysiert werden (erstes Jobinterview, zweites Jobinterview und Jobangebot; diese Stufen werden zum Teil noch weiter differenziert). Yakubovich / Lup (2006) nehmen Bewerbungen für eine Position als ,sales agent“ in einem US-amerikanischen ,virtual call center“ in den Blick. Hierbei stehen fünf Rekrutierungsstufen im Mittelpunkt (u.a. Tests, Jobangebot, Trainingsstufe).

Jede dieser Studien stammt demnach aus den USA und drei der fünf Studien konzentrieren sich explizit und ausschließlich auf Einstiegsjobs. Darüber hinaus unterscheiden sich die Stu-

6 Es ist nicht auszuschließen, dass Vermittler in ihren Handlungen mitunter kurzfristig orientiert sind und versuchen, möglichst viele Unternehmen zu bedienen, und dabei eine mangelnde Eignung von Jobkandidaten in Kauf nehmen. In der Praxis werden sicherlich auch deshalb oftmals anreizorientierte Verträge mit Headhuntern geschlossen, die beispielsweise einen gewissen Prozentsatz der Zahlungssumme bei Zustandekommen eines Arbeitsvertrages garantieren und den Restsatz erst dann, wenn der Kandidat die Probezeit im Unternehmen überstanden hat. 
dien in ihren spezifischen Fragestellungen, etwa durch einen Fokus auf die ethnische Herkunft der Bewerber. Alle Studien verdeutlichen aber zunächst deskriptiv, welche beachtlichen Anteile soziale Kontakte bzw. Belegschaftsempfehlungen am Bewerberpool ausmachen. Soziale Kontakte erlangen am Gesamtbewerberpool Anteile von $8 \%$ in der Studie von Fernandez/ Weinberg (1997) bis zu 51\% in der Studie von Petersen et al. (2000). Außer in der Studie von Yakubovich / Lup (2006) erhalten Mitarbeiter, die einen erfolgreichen Kandidaten empfohlen haben, zudem eine Prämie (z.B. in Fernandez / Sosa 2005 \$10, wenn der Kandidat interviewt wurde, sowie \$250, wenn der Kandidat die Probezeit überstanden hat).

In den multivariaten Analysen, unter Kontrolle wichtiger Variablen wie Humankapital, wird sichtbar, dass sich die Rekrutierung über soziale Kontakte auf den einzelnen Rekrutierungsstufen im Vergleich zu Rekrutierungen auf dem formellen Weg (Anzeigen usw.) ganz überwiegend auszahlt. Humankapitalfaktoren können diese Vorteile sozialer Kontakte kaum erklären (z.B. bei Fernandez / Weinberg 1997). Belegschaftsempfehlungen erhöhen die Wahrscheinlichkeit, die einzelnen Stufen erfolgreich zu durchlaufen und letztendlich ein Jobangebot zu bekommen. Bei Petersen et al. (2000) sind solche direkten Effekte der Rekrutierungsstrategie lediglich auf der ersten Rekrutierungsstufe relevant. Auf allen weiteren Stufen liegen aber immer noch über die erste Stufe vermittelte indirekte Effekte vor. Diese Untersuchung betrachtet als einzige der fünf Studien in Tabelle 1 die Bedeutung von Headhuntern als Rekrutierungskanal und zeigt, dass Headhunter-Kandidaten, vergleichbar mit sozialen Kontakten, eine höhere Erfolgswahrscheinlichkeit im Rekrutierungsprozess haben. 


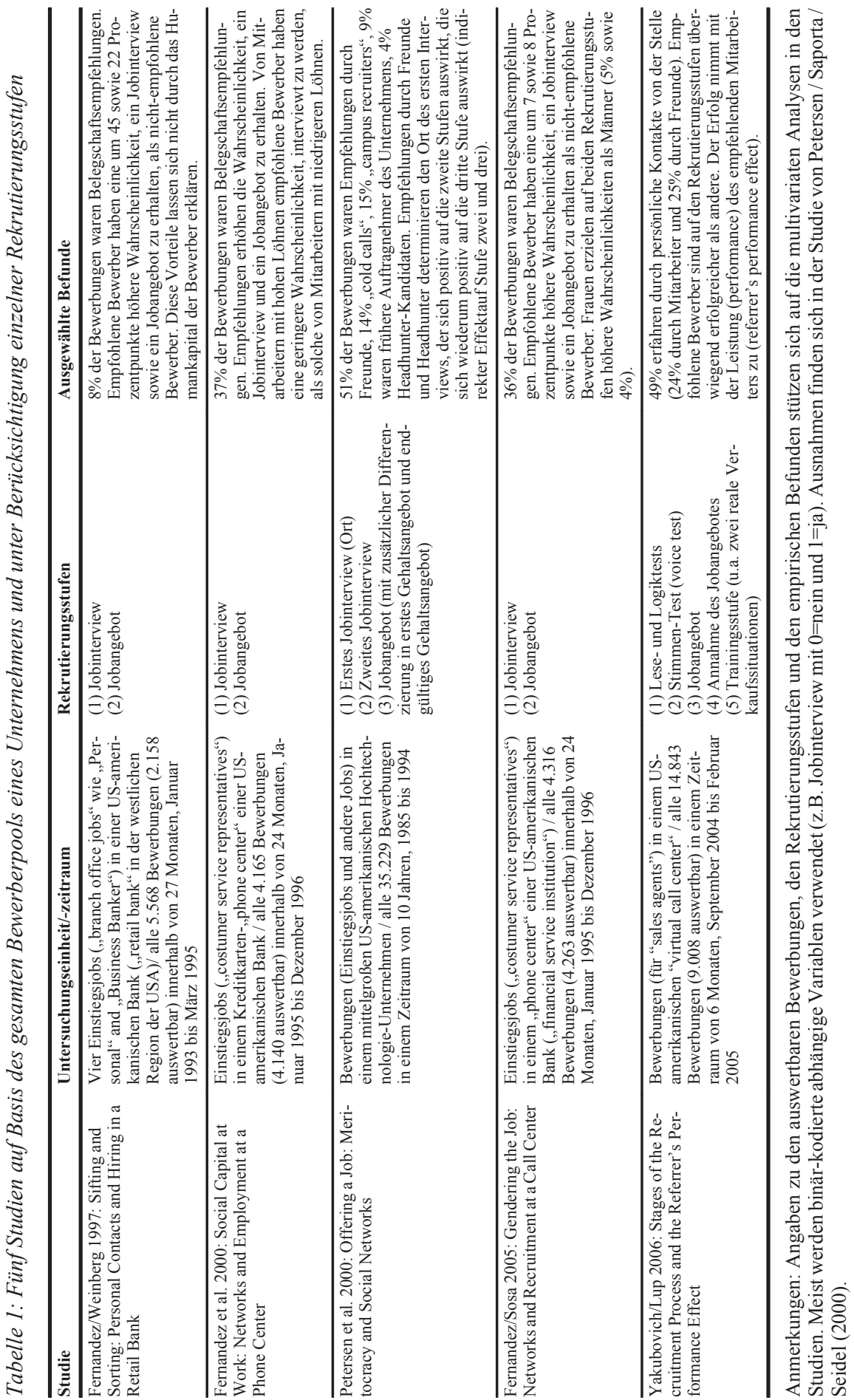


Weitere interessante Befunde in den Studien sind beispielsweise bei Fernandez / Weinberg (1997), dass im Vergleich zu „,formellen Bewerbern“ empfohlene Bewerber Lebensläufe haben, die besser die Unternehmensanforderungen erfüllen, und empfohlene Bewerber aufgrund ihrer spezifischen Informationen eher geeignete Zeitpunkte wählen, ihre Bewerbung einzureichen. Fernandez et al. (2000) finden entgegen der theoretischen Erwartungen, dass im Vergleich zu nicht empfohlenen Mitarbeitern empfohlene Mitarbeiter keine besseren Informationen über die Stelle und keine geringere Fluktuationsquote haben. Ebenso haben auch die rekrutierenden Personen keine besseren Informationen über die empfohlenen Bewerber. In der Studie von Petersen et al. (2000) verliert die ethnische Herkunft der Bewerber bei Kontrolle der Rekrutierungskanäle an Bedeutung (Aufhebung ethnischer Diskriminierung). Jedoch haben ethnische Minderheiten ex ante einen schlechteren Zugang zu hilfreichen sozialen Netzwerken oder aber sie nutzen ihre Netzwerke nicht entsprechend. Ferner führen in dieser Studie Empfehlungen durch Freunde und Headhunter zu höheren Gehältern und einer geringeren Fluktuation (innerhalb eines Jahres nach Aufnahme des Jobs).

Fernandez / Sosa (2005) zeigen zum einen, dass neben Belegschaftsempfehlungen auch unternehmensinterne Bewerber im Rekrutierungsprozess besonders erfolgreich sind, und zum anderen, dass Frauen bei Kontrolle wichtiger Variablen auf allen Rekrutierungsstufen erfolgreicher abschneiden als Männer (wobei laut den Autoren bei einem Job in einem ,phone center“" die rekrutierenden Personen Frauen mitunter bewusst oder unbewusst bevorzugen). Ein Kernbefund von Yakubovich / Lup (2006) ist, dass auf fast allen Rekrutierungsstufen die objektive Überlegenheit (Leistungen) und die wahrgenommenen Fähigkeiten der empfohlenen Bewerber mit der Leistung des Empfehlenden im eigenen Unternehmen zunehmen (referrer's performance effect).

Insgesamt deuten die betrachteten Studien darauf hin, dass soziale Kontakte bzw. Belegschaftsempfehlungen die Erfolgswahrscheinlichkeit im Rekrutierungsprozess erhöhen. Allerdings legen die Studien nahe, dass nicht alle in Abschnitt 2.1 postulierten sozialen Mechanismen durchweg bestätigt werden. Dies betrifft vor allem die Erwartung, dass empfohlene Bewerber nach der Einstellung eine höhere Leistung erbringen sowie eine geringere Fluktuation aufweisen als formelle Bewerber. Mit Blick auf die Bedeutung von Headhuntern liegt lediglich eine Studie vor. Zusammengenommen besteht ein deutlicher Bedarf an weiteren empirischen Untersuchungen.

\section{Daten und beschreibende Analysen}

Im Folgenden werden die von uns verwendeten Daten beschrieben. In einem ersten Schritt charakterisieren wir das untersuchte Unternehmen sowie dessen Rekrutierungsstrategie und gehen auf die Datenerhebung näher ein. Beschreibende Analysen zum Bewerberpool, zu den Rekrutierungskanälen und zum Erfolg auf den einzelnen Stufen im Rekrutierungsprozess folgen in einem zweiten Schritt.

\subsection{Daten}

Die empirische Datenbasis bezieht sich auf das Rekrutierungsgeschehen im Zeitraum von Februar bis April 2007 in einem international agierenden Unternehmen aus der Textil-Branche (Online-Zusammenstellung und -Vertrieb von Textilprodukten aus der eigenen Herstellung), das seinen Hauptsitz (seit der Gründung 2003) in den neuen Bundesländern hat. Dieses Unternehmen befand sich im Untersuchungszeitraum in einer Phase des organisationalen Wandels vom typischen Start-Up-Unternehmen mit einem geringen Organisationsgrad und wenig geregelten Abläufen hin zu einem Unternehmen mit gefestigten Organisationsstrukturen und -prozessen. Im Zuge dieses Wandels wurde auch eine Personalabteilung mit drei Mitarbeitern eingerichtet. Zum Zeitpunkt der Datenerhebung hatte das Unternehmen weltweit ca. 250 Mit- 
arbeiter (davon ca. 90 Mitarbeiter am Hauptsitz) und aufgrund einer Wachstumsphase einen starken Personalbedarf. Dieser Bedarf bezog sich auf verschiedene Positionen im Unternehmen, die durch unterschiedliche Qualifikationsanforderungen gekennzeichnet waren. Offene Stellen wurden in den Bereichen Unternehmensführung, Verwaltung (z.B. Buchhaltung, Personal- und Rechtsabteilung), Grafik, Marketing und Vertrieb, Kundenservice, IT (,,information technology") sowie im Produktionsbereich (z.B. leitende Angestellte) besetzt.

Mit Blick auf die Mitarbeiterrekrutierung wurden möglichst genaue Bewerberprofile erarbeitet und eine zielgerichtete Bewerbersuche angestrebt. Neben kostenfreien Online-Portalen, Aushängen an Hochschulen sowie persönlichen Netzwerken wurden auch kostenpflichtige Internet-Stellenbörsen, Anzeigen in lokalen Print-Medien, Werbung auf Karriere-Messen und mitunter (bei besonders spezialisierten Positionen) Headhunter genutzt. Vergleichbar mit der Studie von Yakubovich / Lup (2006) hat das hier betrachtete Unternehmen Belegschaftsempfehlungen nicht aktiv durch Prämien belohnt, d.h. es liegen primär informelle Prozesse bei der Rekrutierung über soziale Kontakte vor (Belegschaftsempfehlungen als passiver Rekrutierungskanal).

Im Untersuchungszeitraum vollzog sich die Mitarbeiterrekrutierung in einem dreistufigen Prozess. Auf der ersten Stufe wurden alle Bewerbungen durch einen Mitarbeiter der Personalabteilung gesichtet, vorselektiert und den einzelnen Fachabteilungen zugeordnet. Die Auswahlkriterien waren hier zum einen formale Ansprüche an die Bewerbungsunterlagen, zum anderen wurden unpassende Bewerberprofile abgelehnt. Eine Überprüfung der verbleibenden Unterlagen durch einen Vertreter der Fachabteilung erfolgte auf der zweiten Stufe. Neben formellen Qualifikationen wurden hier auch fachspezifische Bewerbermerkmale in Augenschein genommen. Hierzu gehörten beispielsweise die bisherigen Berufsstationen eines Bewerbers. War eine Bewerbung auf dieser Auswahlstufe erfolgreich, folgten auf der dritten Stufe Vorstellungsgespräche. Je nach den Stellenanforderungen handelte es sich hierbei um ein Telefoninterview sowie ein oder mehrere persönliche Gespräche mit Verantwortlichen der einstellenden Abteilung. Bei den Gesprächen wurde neben tiefergehenden fachlichen Fragen vor allem die persönliche Eignung der Kandidaten überprüft. Dabei achtete das Unternehmen vor allem auf ,soft skills“ (u.a. die Fähigkeit, selbständig Innovationen voranzutreiben sowie Aufgaben pragmatisch zu lösen). Auf standardisierte Auswahlverfahren wie ein Assessment-Center oder Leistungstests wurde bei der Bewerberauswahl verzichtet. Erst nach der erfolgreichen Absolvierung aller drei Stufen wurde den Bewerbern ein Arbeitsvertrag angeboten. Der Auswahlprozess war insgesamt so strukturiert, dass keine Vorteile für solche Bewerber zu erwarten sind, die über soziale Kontakte oder über Headhunter rekrutiert wurden. Auch diese Personen mussten alle drei Stufen im Rekrutierungsprozess durchlaufen.

Die Datengrundlage bilden 477 Bewerbungen, die im untersuchten Unternehmen im Zeitraum vom 1.2.2007 bis zum 30.4.2007 eingegangen sind. Datenquellen sind Unternehmensaufzeichnungen und die einzelnen Bewerbungsunterlagen/-mappen (zur Ermittlung zentraler Merkmale wie Bildungsabschlüsse und Branchenerfahrung). Jede Bewerbung wurde von einem Mitarbeiter der Personalabteilung in eine Datenbank eingepflegt. Neben den Kontaktinformationen und dem Namen des Bewerbers wurden auch das Eingangsdatum der Bewerbung, die angestrebte Stelle oder Abteilung, die Abteilung, an welche die Bewerbung zur Prüfung weitergeleitet wurde, gegebenenfalls Interviewtermine sowie der Status der Bewerbung erfasst. Die Angaben zur prüfenden Abteilung und zu den Interviewterminen dienten als Grundlage, um die unterschiedlichen Stufen im Rekrutierungsprozess abzubilden. Wurde eine Bewerbung nicht zur Prüfung an eine Abteilung weitergeleitet, bedeutete dies eine Absage auf dieser Auswahlstufe. Waren keine Interviewtermine verzeichnet, wurde dem Bewerber nach der Prüfung seiner Unterlagen durch die Fachabteilung abgesagt. Zum Zeitpunkt der Datenerhebung konnten alle Bewerbungen einem der folgenden drei Endstadien zugeordnet werden: 
(1) das Unternehmen sagt auf einer der drei Rekrutierungsstufen dem Bewerber ab, (2) der Bewerber sagt auf einer der drei Rekrutierungsstufen dem Unternehmen ab, oder aber (3) das Unternehmen und der Bewerber schließen einen Arbeitsvertrag.

\subsection{Beschreibende Analysen}

Gemäß den drei Stufen im Rekrutierungsprozess wird im Folgenden dargelegt, welche Bewerberanteile auf den einzelnen Stufen erfolgreich gewesen sind. Tabelle 2 gibt hierzu einen Überblick und liefert weitere deskriptive Informationen zu den drei Bereichen Humankapital, Rekrutierungskanäle und Kontrollvariablen, die zudem die Variablenbasis für die multivariaten Analysen in Abschnitt 4 bilden. Dabei werden Fälle mit fehlenden Werten ausgeschlossen. Somit reduziert sich die Gesamtdatenbasis von 477 auf 441 Fälle. „Erfolg“ auf einer der drei Rekrutierungsstufen ist wie folgt definiert: Erfolgreich ist ein Bewerber jeweils, wenn seine Bewerbung an eine Fachabteilung weitergeleitet wurde, sie/er zum Jobinterview eingeladen wurde und ein Jobangebot erhalten hat, unabhängig davon, ob sie/er eine Einladung zum Jobinterview oder das Jobangebot abgelehnt hat. Deskriptive Werte für Bewerberabsagen als Teilgruppe der erfolgreichen Bewerber werden dann nochmals gesondert ausgewiesen.

Zunächst wird in Tabelle 2 ersichtlich, dass von den insgesamt 477 Bewerbungen $81 \%$ die erste Hürde genommen haben und somit an die jeweilige Fachabteilung weitergeleitet wurden. Von diesen 384 Bewerbern wurden 34\% zum Interview eingeladen und von diesen 132 Bewerbern wiederum $34 \%$ ein Jobangebot unterbereitet. Darüber hinaus bleibt zu erwähnen, dass auf der Interviewstufe 8 von 384 Bewerbern (2\%) und auf der Jobangebotsstufe 23 von 132 Bewerbern (17\%) dem Unternehmen von sich aus abgesagt haben. Letztendlich wurden 22 Bewerber (knapp 5\%) von insgesamt 477 Bewerbern eingestellt, d.h. hier haben beide Seiten, das Unternehmen und der Bewerber, eine Einigung erzielt. ${ }^{7}$

7 Bei der Interviewstufe und Einstellungsentscheidung kann davon ausgegangen werden, dass Bewerberabsagen zu „Auffüllungen“ geführt haben, d.h. nach einer Absage eines Bewerbers ein anderer Bewerber zum Zuge gekommen ist. Dieser sequenzielle Prozess kann mit den vorliegenden Daten nicht gesondert abgebildet werden. 
Tabelle 2: Gesamtschau der abhängigen und unabhängigen Variablen

\begin{tabular}{|c|c|c|c|}
\hline \multirow[t]{2}{*}{ Variable } & $\begin{array}{l}\text { Weiterleitung an Fach- } \\
\text { abteilung }\end{array}$ & $\begin{array}{l}\text { Einladung zum Inter- } \\
\text { view }\end{array}$ & Jobangebot \\
\hline & Mittelwert (Stdabw) & Mittelwert (Stdabw) & Mittelwert (Stdabw) \\
\hline $\begin{array}{l}\text { Abhängige Variablen } \\
\text { Fachabteilung }(1=\mathrm{ja})\end{array}$ & $\begin{array}{l}0,81(0,39) \\
{[0,81(0,40)]}\end{array}$ & & \\
\hline Interview $(1=\mathrm{ja})$ & & $\begin{array}{l}0,35(0,48) \\
{[0,34(0,48)]}\end{array}$ & \\
\hline Jobvergabe $(1=\mathrm{ja})$ & & & $\begin{array}{l}0,31(0,47) \\
{[0,34(0,48)]}\end{array}$ \\
\hline $\begin{array}{l}\text { Unabhängige Variablen } \\
\text { Humankapital } \\
\text { Bildung (1=Fachhochschule/ } \\
\text { Universität) }\end{array}$ & $0,60(0,49)$ & $0,66(0,48)$ & $0,75(0,43)$ \\
\hline Branchenerfahrung (1=ja) & $0,08(0,27)$ & $0,09(0,29)$ & $0,21(0,41)$ \\
\hline $\begin{array}{l}\text { Rekrutierungskanäle } \\
\text { (formelle/andere Kanäle als Refe- } \\
\text { renz) } \\
\text { Headhunter }(1=\text { ja) }\end{array}$ & $0,12(0,33)$ & $0,15(0,36)$ & $0,31(0,47)$ \\
\hline Empfehlungen/soz. Kontakte (1=ja) & $0,03(0,18)$ & $0,04(0,19)$ & $0,08(0,27)$ \\
\hline $\begin{array}{l}\text { Kontrollvariablen } \\
\text { Geschlecht (1=Frau) }\end{array}$ & $0,33(0,47)$ & $0,32(0,47)$ & $0,30(0,46)$ \\
\hline Alter in Jahren & $32,07(8,25)$ & $32,36(7,80)$ & $32,18(6,43)$ \\
\hline $\begin{array}{l}\text { Angestrebte Abteilung } \\
\text { (keine Angabe als Referenz) } \\
\text { Vorstand }(1=\mathrm{ja})\end{array}$ & $0,06(0,24)$ & $0,07(0,48)$ & $0,14(0,47)$ \\
\hline Verwaltung $(1=\mathrm{ja})$ & $0,12(0,32)$ & $0,13(0,34)$ & $0,17(0,38)$ \\
\hline Technik/IT (1=ja) & $0,49(0,50)$ & $0,53(0,50)$ & $0,50(0,50)$ \\
\hline Kundenbetreuung $(1=\mathrm{ja})$ & $0,22(0,41)$ & $0,23(0,42)$ & $0,18(0,38)$ \\
\hline $\begin{array}{l}\text { Bewerbungsmonat (Februar als } \\
\text { Referenz) } \\
\text { März }(1=\mathrm{ja}) \\
\text { April }(1=\mathrm{ja})\end{array}$ & $\begin{array}{l}0,29(0,46) \\
0,31(0,46)\end{array}$ & $\begin{array}{l}0,27(0,45) \\
0,33(0,47)\end{array}$ & $\begin{array}{l}0,30(0,46) \\
0,42(0,50)\end{array}$ \\
\hline $\mathrm{N}$ & $\begin{array}{l}441 \\
{[477]}\end{array}$ & $\begin{array}{l}358 \\
{[384]}\end{array}$ & $\begin{array}{l}125 \\
{[132]}\end{array}$ \\
\hline
\end{tabular}

Anmerkungen: Die Angaben und Fallzahlen in den eckigen Klammern [...] beziehen sich auf verfügbare Fälle ohne Ausschluss von Fällen mit fehlenden Werten. Auf der Interviewstufe haben 8 von 358 Bewerbern (2\%) bzw. 8 von 384 Bewerbern [2\%] und auf der Jobvergabestufe 20 von 125 Bewerbern (16\%) bzw. 23 von 132 Bewerbern [17\%] dem Unternehmen von sich aus abgesagt. Letztendlich wurden 19 von 125 Bewerbern (5\%) bzw. 22 von 132 Bewerbern [5\%] eingestellt.

Mit Blick auf die reduzierte Datenbasis und die abhängigen Variablen werden in Tabelle 2 geringfügige Änderungen ersichtlich. Auf der ersten Rekrutierungsstufe werden $81 \%$ der eingegangenen Bewerbungen an eine entsprechende Fachabteilung weitergeleitet, auf der zweiten Stufe werden $35 \%$ der Bewerber zum Interview eingeladen, von denen schließlich auf der dritten Stufe 31\% ein Jobangebot erhalten. Als Humankapitalvariablen werden der Bildungsgrad und die relevante Branchenerfahrung der Bewerber herangezogen. Bildung geht als binär kodierte Variable in die Analysen ein, deren Wert Null ist, wenn der Bewerber keinen Schulabschluss, einen Volks- / Hauptschulabschluss, eine mittlere Reife / Realschulabschluss, eine Fachhochschulreife oder aber ein Abitur besitzt, und den Wert Eins annimmt, wenn der Bewerber einen Fachhochschulabschluss oder einen Universitätsabschluss hat. Dieser Anteil nimmt über die Rekrutierungsstufen hinweg zu (von 60\% auf 75\%). Ob ein Bewerber über Branchenerfahrung im Internet- oder eCommerce-Bereich verfügt, wurde unmittelbar den Be- 
werbungsunterlagen entnommen, d.h. es wurde ermittelt, inwieweit im Lebenslauf solche Erfahrungen kenntlich gemacht wurden (durch entsprechende Berufsstationen / -positionen). Der Anteil an Bewerbern mit Branchenerfahrung steigt im Zuge des Rekrutierungsprozesses an, besonders deutlich, wenn es um die Entscheidung über ein Jobangebot geht (von $8 \%$ über 9\% auf schließlich 21\%).

Interessant ist der Blick auf die verwendeten Rekrutierungskanäle, die den Bewerbungsunterlagen entnommen wurden. Dabei unterscheiden wir zwischen Headhuntern und dem Verweis auf persönliche Kontakte auf der einen Seite und formellen sowie anderen Rekrutierungskanälen (als Referenzkategorie) auf der anderen Seite. Zu den formellen und anderen Rekrutierungskanälen zählen Verweise im Bewerbungsschreiben auf Internetrecherchen, Zeitungsanzeigen, Medienberichte, die Firmenhomepage, Aushänge an Hochschulen, Messen, das Arbeitsamt sowie Initiativbewerbungen und Bewerbungen ohne Hinweis auf den Rekrutierungskanal. Headhunter bedeutet, dass das Unternehmen spezialisierte Personalvermittlungen beauftragt hat, geeignete Führungskräfte und hoch qualifizierte Spezialisten (z.B. ITFachkräfte) zu finden. Der Anteil derart motivierter Bewerbungen liegt auf der ersten Stufe bei 12\%, auf der zweiten Stufe bei 15\% und auf der dritten Stufe bei 31\%. Damit scheint sich zunächst ein gewisser Erfolg dieser Rekrutierungsstrategie deskriptiv abzuzeichnen.

Bei persönlichen bzw. sozialen Kontakten (Belegschaftsempfehlungen) wurde ermittelt, ob ein Bewerber in seinem Bewerbungsschreiben direkt auf persönliche Kontakte zu einem Mitarbeiter im Unternehmen verwiesen hat. Dabei kann es sich um private Kontakte, d.h. der Bewerber ist z.B. ein Verwandter oder Freund des Mitarbeiters im Unternehmen, oder aber um berufliche Kontakte handeln, d.h. der Bewerber hat eine arbeitsbezogene Beziehung zum Mitarbeiter des Unternehmens. Der Anteil von Bewerben, die sich in ihrer Bewerbung auf solche Kontakte beziehen, beträgt auf den ersten beiden Stufen 3\% bzw. 4\% und auf der dritten Stufe $8 \%$. Damit ist bezüglich eines Jobangebotes zumindest deskriptiv eine Tendenz sichtbar, dass sich soziale Kontakte im Rekrutierungsprozess auszahlen. Im Vergleich zu den Studien in Abschnitt 2.2 liegt in der vorliegenden Untersuchung ein vergleichsweise geringer Anteil an Mitarbeiterempfehlungen vor. Es kann nicht ausgeschlossen werden, dass dieser Anteil unterschätzt wird.

Eine Begründung liefert Tabelle 3, die die Bewerberanteile auf jeder Rekrutierungsstufe für einzelne Rekrutierungskanäle detailreicher berichtet. Unterschieden werden formelle Rekrutierungen (sowie Initiativbewerbungen), Belegschaftsempfehlungen, Headhunter und solche Bewerbungen, die keinem Rekrutierungskanal zugeordnet werden konnten (,keine Angabe“). Letztere machen mit 16\% einen beachtlichen Anteil aus. Der höchste Bewerberanteil auf der ersten Stufe ergibt sich für formelle Rekrutierungen, gefolgt von „,keine Angabe“, Headhunter und Empfehlungen (bzw. sozialen Kontakten). Auf der einen Seite ist es möglich, dass insbesondere bei „keine Angabe“ auch die Nutzung sozialer Kontakte verborgen bleibt, wobei dies aber gleichermaßen auch auf alle anderen Rekrutierungskanäle zutrifft. Auf der anderen Seite erlaubt unser Vorgehen ein „konservatives“ Maß für die informelle Rekrutierung über soziale Kontakte, da durch die Benennung des Mitarbeiters in den Bewerbungsunterlagen des Bewerbers, der jeweilige Mitarbeiter auch, in die Pflicht genommen wird“, ganz im Sinne der theoretisch postulierten Mechanismen in Abschnitt 2.1 (z.B. mit Blick auf die Reputation des benannten Mitarbeiters im eigenen Unternehmen). Entgegen den Erwartungen zeigt Tabelle 3 auch, dass Headhunter-Kandidaten vergleichsweise schlecht abschneiden, wenn es um ein tatsächliches Jobangebot geht (,Jobangebot erfolgt“). Rekrutierungen über soziale Kontakte wiederum erhalten verhältnismäßig häufig ein Jobangebot, wobei hierbei die geringen Fall- 
zahlen einschränkend zu berücksichtigen sind. ${ }^{8}$ Wir haben uns schließlich dafür entschieden, formelle Rekrutierungskanäle (inklusive Initiativbewerbungen) und „keine Angabe“ zu einer Gruppe zusammenzufassen. Dieses Vorgehen hat keine nennenswerten Auswirkungen in den multivariaten Analysen in Abschnitt 4 (d.h. es gibt keine gravierenden Effekte für spezifische Untergruppen).

\section{Tabelle 3: Bewerberanteile auf jeder Rekrutierungsstufe per Rekrutierungskanal in Prozent}

\begin{tabular}{|c|c|c|c|c|c|c|c|c|}
\hline \multirow{2}{*}{$\begin{array}{l}\text { Rekrutierungskanal } \\
\text { Formeller Kanal }\end{array}$} & \multicolumn{2}{|c|}{$\begin{array}{l}\text { Weiterleitung an } \\
\text { Fachabteilung }\end{array}$} & \multicolumn{2}{|c|}{$\begin{array}{l}\text { Einladung } \\
\text { zum Interview }\end{array}$} & \multicolumn{2}{|c|}{ Jobangebot } & \multicolumn{2}{|c|}{$\begin{array}{l}\text { I Jobangebot } \\
\text { I erfolgt }\end{array}$} \\
\hline & 69 & $(302)$ & 68 & $(245)$ & 48 & $(60)$ & 49 & (19) \\
\hline Empfehlungen/soz. Kontakte & 3 & $(15)$ & 4 & (14) & 8 & (10) & 15 & (6) \\
\hline Headhunter & 12 & $(55)$ & 15 & (53) & 31 & (39) & 21 & (8) \\
\hline Keine Angabe & 16 & (69) & 13 & (46) & 13 & (16) & 15 & (6) \\
\hline Gesamt & 100 & $(441)$ & $\begin{array}{l}10 \\
0\end{array}$ & $(358)$ & 100 & $(125)$ & $\begin{array}{l}10 \\
0\end{array}$ & (39) \\
\hline
\end{tabular}

Anmerkungen: Absolute Häufigkeiten in Klammern. Die Gesamtdatenbasis umfasst 441 Bewerber. Die Anteile beziehen sich auf die Datenbasis zu jeder Rekrutierungsstufe. „Jobangebot erfolgt“ betrachtet die Bewerber, die letztendlich ein Jobangebot erhalten haben. Aufgrund der geringen Fallzahl muss vor allem in der letzten Spalte das Augenmerk auf die absoluten Häufigkeiten und nicht auf die Prozentangaben gerichtet werden.

Den Kontrollvariablen in Tabelle 2 lässt sich entnehmen, dass ca. 30 Prozent der Bewerber auf den einzelnen Rekrutierungsstufen weiblich sind und das Durchschnittsalter der Bewerber bei jeweils 32 Jahren liegt. Die Altersvariable hat aber eine weitere Bedeutung. Sie korreliert deutlich mit der Berufserfahrung der Jobkandidaten (gemessen an der Anzahl der Berufsjahre) und mit der Anzahl der Jobwechsel der Bewerber (Pearson'sche Korrelation von 0,863 sowie $0,663$ mit jeweils $\mathrm{p}<0,001)$. Deshalb kann die Altersvariable in den multivariaten Analysen als Proxy für die beiden Variablen Berufserfahrung und Anzahl der Jobwechsel angesehen werden. Eine weitere Kontrollvariable ist die angestrebte Abteilung der Bewerber. Die Fallzahlen lassen es mit Einschränkungen zu, für einzelne „Zielabteilungen“ getrennte Analysen vorzunehmen. Wir unterscheiden zwischen Vorstand (Geschäftsführung), Verwaltung (Buchhaltung, Personal- und Rechtsabteilung), Technik/IT (Produktion, Programmierung, Systembetrieb etc.), Kundenbetreuung (Vertrieb, Marketing und Kundenservice) sowie „keine Angabe“ (Bewerbungen, die keine Angaben zur angestrebten Abteilung gemacht haben). ${ }^{9}$ In Tabelle 2 wird sichtbar, dass die Anteile der Jobkandidaten, die eine nicht-technische und technische Abteilung anstreben, recht ausgeglichen sind. Zudem nehmen die Anteile in den Bereichen Vorstand und Verwaltung über die einzelnen Stufen hinweg zu, während sie im Bereich der Kundenbetreuung abnehmen. Die letzte Kontrollvariable bildet der Bewerbungszeitpunkt,

8 Für die Gruppe der Bewerberabsagen wird darüber hinaus deutlich, dass 9 Absagen aus der Gruppe „formelle Kanäle“, 6 Absagen aus der Gruppe „Headhunter“, 4 Absagen aus der Gruppe „,keine Angabe“ und 1 Absage aus der Gruppe „Empfehlungen/persönliche Kontakte“ stammen, was unter einer grob-fahrlässigen Vernachlässigung der geringen Fallzahlen ein bemerkenswert hohen Anteil in der Gruppe der Headhunter-Kandidaten ausmacht (6 von 8 Bewerbern, die ein Jobangebot erhalten haben). Dies ist auf Grundlage der theoretischen Ausführungen in Abschnitt 2.1 („,better match“) nicht zu erwarten gewesen.

9 Die Fallzahlen lassen zudem keine getrennte Betrachtung von privaten und beruflichen Kontakten zu. Deskriptiv zeichnet sich jedoch ab, dass Bewerbungen über berufliche Kontakte eher eine nicht-technische Tätigkeit im Unternehmen anstreben. Bei privaten Kontakten liegt eine annähernde Gleichverteilung auf nicht-technische und technische Abteilungen vor. Dies trifft auch auf Headhunter-Kandidaten zu und ist hierbei plausibel, da in beiden Bereichen (nicht-technische und technische Abteilungen) u.a. Spezialisten gesucht wurden. 
der durch den Bewerbungsmonat erfasst wird. Auf den einzelnen Rekrutierungsstufen steigt insbesondere der Anteil der Bewerber im Monat April.

\section{Ergebnisse der multivariaten Analysen}

Um zu ermitteln, inwieweit die deskriptiven Befunde aus dem vorhergehenden Abschnitt unter Kontrolle wichtiger Variablen bedeutsam bleiben, wurden Probit-Modelle für die einzelnen Rekrutierungsstufen geschätzt. Die Ergebnisse lassen sich Tabelle 4 entnehmen. Diese enthält neben den einzelnen Koeffizienten auch ,durchschnittliche Einheitseffekte“ (,average marginal effects“, von uns \%-Effekt benannt) mit Blick auf die Wahrscheinlichkeit, eine Rekrutierungsstufe erfolgreich zu überstehen, wenn sich die jeweilige Variable (ceteris paribus) um eine Einheit ändert. Dazu wird der Mittelwert über die Einheitseffekte (,,marginal efects“) aller Beobachtungen berechnet.

Tabelle 4: Multivariate Analysen zu den einzelnen Rekrutierungsstufen (Probit-Modelle)

\begin{tabular}{|c|c|c|c|c|c|c|}
\hline & Fachabteilung & $\%$-Effekt & Interview & $\%$-Effekt & $\begin{array}{l}\text { Jobange- } \\
\text { bot }\end{array}$ & $\%$-Effekt \\
\hline \multicolumn{7}{|l|}{ Humankapital } \\
\hline Bildung ( $1=\mathrm{FH} /$ Universität $)$ & $\begin{array}{l}0,56^{*} \\
(3,16)\end{array}$ & 0,10 & $\begin{array}{l}0,45^{*} \\
(2,61)\end{array}$ & 0,13 & $\begin{array}{l}-0,12 \\
(-0,41)\end{array}$ & $-0,04$ \\
\hline Branchenerfahrung (1=ja) & $\begin{array}{l}1,46^{*} \\
(2,64)\end{array}$ & 0,15 & $\begin{array}{l}1,19^{*} \\
(4,02)\end{array}$ & 0,38 & $\begin{array}{l}0,26 \\
(0,72)\end{array}$ & 0,09 \\
\hline \multicolumn{7}{|c|}{$\begin{array}{l}\text { Rekrutierungskanäle (formelle/ an- } \\
\text { dere Kanäle als Referenz) }\end{array}$} \\
\hline Headhunter $(1=\mathrm{ja})$ & $\begin{array}{l}0,82 * \\
(2,44)\end{array}$ & 0,11 & $\begin{array}{l}0,81 * \\
(3,51)\end{array}$ & 0,26 & $\begin{array}{l}-0,64 \\
(-1,91)\end{array}$ & $-0,18$ \\
\hline $\begin{array}{l}\text { Empfehlungen/soz. Kontakte } \\
(1=\text { ja) }\end{array}$ & $\begin{array}{l}1,76^{*} \\
(2,58)\end{array}$ & 0,16 & $\begin{array}{l}1,21^{*} \\
(2,95)\end{array}$ & 0,39 & $\begin{array}{l}0,79 \\
(1,76)\end{array}$ & 0,28 \\
\hline Pseudo-R ${ }^{2}$ (McFadden) & 0,319 & & 0,186 & & 0,079 & \\
\hline $\mathrm{N}$ & 441 & & 358 & & 125 & \\
\hline
\end{tabular}

Anmerkungen: * signifikant auf dem 5\%-Niveau; Schätzungen mit robusten Standardfehlern (,HuberWhite-Sandwich-Schätzer"); z-Werte in Klammern. Alle Modelle enthalten die Kontrollvariablen Geschlecht, Alter in Jahren, die angestrebte Abteilung und den Bewerbungsmonat. Im Unterschied zum Fachabteilungs- und Interviewmodell weist das Jobangebotsmodell keine statistische Signifikanz auf. „\%Effekt" bezieht sich auf die „durchschnittlichen marginalen Effekte“ (,average marginal effects“): den Mittelwert über die marginalen Effekte für jede Beobachtung.

Für die erste Rekrutierungsstufe, die Weitergabe der Bewerbung an eine Fachabteilung, zeigen die Ergebnisse in Tabelle 4, dass sowohl das Humankapital als auch der Rekrutierungskanal von Bedeutung sind. Bewerber mit einem Fachhochschulabschluss (FH) oder Universitätsabschluss und mit relevanter Branchenerfahrung haben eine höhere Wahrscheinlichkeit, dass ihre Bewerbung an die Fachabteilung weitergegeben wird als Bewerber ohne diese höheren Bildungsabschlüsse und ohne Branchenerfahrung. Im Vergleich zu formellen Rekrutierungskanälen (und Bewerbungen ohne Angabe des Kanals) ergeben sich für HeadhunterBewerber und empfohlene Bewerber zudem die erwarteten positiven Effekte auf der ersten Stufe. Diese Befunde illustrieren nochmals die Einheits- bzw. Prozenteffekte. Im Durchschnitt erhöht sich die Wahrscheinlichkeit, die erste Stufe erfolgreich zu absolvieren, jeweils um 10 sowie 15 Prozentpunkte, wenn der Bewerber mindestens einen FH-Abschluss sowie Branchenerfahrung hat, um 11 sowie 16 Prozentpunkte für über Headhunter sowie soziale Kontakte aktivierte Bewerbungen.

Auf der zweiten Rekrutierungsstufe, die Einladung zu einem Jobinterview, ergibt sich ein ähnliches Bild wie auf der ersten Stufe. Bewerber mit einer höheren Bildung, relevanter Bran- 
chenerfahrung, Headhunter-Bewerber und empfohlene Bewerber haben jeweils eine höhere Wahrscheinlichkeit, zu einem Jobinterview eingeladen zu werden als andere Bewerber. Neben der Bedeutung der Humankapitalausstattung von Bewerbern werden, im Sinne der theoretisch postulierten Mechanismen, auch auf der zweiten Stufe der Informations- und Homophiliemechanismus sichtbar, die einen besseren Match bei empfohlenen Bewerbern und HeadhunterBewerbern gegenüber anderen Bewerbern vermuten lassen. Dies scheint nicht unplausibel, da das Unternehmen nunmehr besonders auf „soft skills“ der Bewerber geachtet hat und diese schwer zu bestimmen sind, sodass dem Rekrutierungskanal mitunter eine „Signalfunktion“ zugesprochen wird. Bewerber mit einer höheren Bildung und relevanter Branchenerfahrung haben im Durchschnitt eine um 13 und 38 Prozentpunkte höhere Interviewwahrscheinlichkeit, Headhunter-Bewerber und empfohlene Bewerber eine um 26 und 39 Prozentpunkte höhere Erfolgswahrscheinlichkeit.

Wenn es um ein Jobangebot geht, die dritte Rekrutierungsstufe, dominieren die Rekrutierungskanäle das Bild. Allerdings ist zu berücksichtigen, dass das Jobangebotsmodell in Tabelle 4 keine statistische Signifikanz aufweist. Jedoch lassen sich die darin angedeuteten zentralen Befunde (schwache Signifikanzen für die Rekrutierungskanäle) zumindest bivariat abstützen, d.h. im Unterschied zu den Humankapitalvariablen liegen bei den Rekrutierungskanälen immerhin (schwach) signifikante bivariate Zusammenhänge vor (Chi-Quadrat-Werte von 4,13 sowie 3,47 und Phi-Koeffizienten von -0,179 sowie 0,164 für Headhunter-Rekrutierungen und Belegschaftsempfehlungen). Wider Erwarten haben Headhunter-Kandidaten eine signifikant geringere Erfolgswahrscheinlichkeit im Vergleich zu formellen Rekrutierungen. Empfohlene Bewerbungen haben den erwarteten positiven Effekt. Einerseits zeigen persönliche Kontakte gegenüber formellen (und anderen) Bewerbungen Vorteile auf der letzten Stufe im Rekrutierungsprozess. Im Vergleich zu formellen Bewerbern haben empfohlene Bewerber eine höhere Wahrscheinlichkeit, ein Jobangebot zu erhalten. Andererseits enthalten die Daten ein „Headhunter-Rätsel“", das sich bereits bei den deskriptiven Analysen angedeutet hat. HeadhunterKandidaten schneiden auf der dritten Rekrutierungsstufe schlechter ab als formelle Bewerber. Zumindest in der vorliegenden Studie bestätigen sich die vermuteten „Better-Match“-Vorteile für Jobangebote nicht. Dieser Befund wird etwas plausibler, falls zum einen im Rahmen des Jobinterviews auch Gehaltsaspekte angesprochen wurden. Es könnte der Fall sein, dass Headhunter-Kandidaten zwar die Unternehmensanforderungen überdurchschnittlich erfüllen, aber besonders hohe Gehaltsvorstellungen haben, wenn sie zudem aus komfortablen Positionen heraus angeworben wurden. Allerdings ist es gerade eine Aufgabe von Headhuntern, solche Punkte zu berücksichtigen. Zum anderen erhält der Headhunter für die Vermittlung erfolgreicher Bewerber eine Prämie. Bei gleicher Eignung von formellen Bewerbern und HeadhunterKandidaten könnte das Unternehmen dazu neigen, eher den formellen Bewerbern ein Jobangebot zu unterbreiten und sich somit die Prämie für den Headhunter „,zu sparen““ ${ }^{10}$

10 Die Kontrollvariablen zeigen in den multivariaten Analysen kein durchweg einheitliches Bild. Zunächst haben, wenig überraschend, Bewerber, die eine Zielabteilung im Unternehmen genannt haben, eine signifikant höhere Wahrscheinlichkeit, die erste Rekrutierungsstufe erfolgreich zu absolvieren, als Bewerber ohne Angabe einer Zielabteilung. Dies trifft überwiegend auch auf die zweite Stufe zu. Zudem kann ein negativer und signifikanter Alterseffekt auf der ersten und zweiten Rekrutierungsstufe berichtet werden (eine Annahme nicht-linearer Alterseffekte wird nicht unterstützt). Damit zahlt sich in der vorliegenden Studie eine zunehmende Berufserfahrung eher nicht aus. Schließlich ergeben sich etwas uneinheitliche Effekte des Bewerbungsmonats. Im Vergleich zum Februar haben Bewerbungen im März eine signifikant geringere Wahrscheinlichkeit, die erste Rekrutierungsstufe zu nehmen, und Bewerbungen im April eine signifikant höhere Wahrscheinlichkeit mit Blick auf die Interviewstufe. 


\section{Schlussbemerkungen}

Obwohl die von uns verwendeten Daten mit einigen Einschränkungen verbunden sind (vor allem mit geringen Fallzahlen für spezifische Untergruppen), bestärkt unser Beitrag die wenigen bisher durchgeführten Studien darin, dass sich für Organisationen die Nutzung sozialer Kontakte bei der Rekrutierung von Mitarbeitern auszahlt. Dies bekräftigt zugleich, so kann argumentiert werden, die Wirkung bestimmter sozialer Mechanismen (z.B. Informations-, Homophilie-, Reputationsmechanismus), die aus einer Organisationsperspektive Vorteile von Belegschaftsempfehlungen gegenüber formellen Rekrutierungskanälen nahe legen (,better match" mit Blick auf die Organisationsanforderungen). Dabei handelt es sich in unserer Studie primär um informelle Prozesse, denn Belegschaftsempfehlungen wurden vom betrachteten Unternehmen nicht aktiv gefördert (z.B. durch eine Prämie). Vielmehr waren soziale Kontakte der Mitarbeiter ein passiver Rekrutierungskanal. Yakubovich / Lup (2006) berichten ähnliche Ergebnisse zur Bedeutung dieser Art von informellen Prozessen bei der Mitarbeiterrekrutierung. Darüber hinaus zeigen unsere Befunde, dass durch Headhunter aktivierte Bewerbungen analog zu sozialen Kontakten zumindest bis zur Entscheidung über die Jobvergabe Vorteile gegenüber formell aktivierten Bewerbern erzielen. Wenn es allerdings um ein Jobangebot bzw. die Einstellungsentscheidung geht, dann schneiden Headhunterkandidaten schlechter ab als formell rekrutierte Bewerber. Petersen et al. (2000) ist die einzige uns bekannte Untersuchung, die auch den Erfolg von Headhunter-Rekrutierungen analysiert. Der von uns berichtete negative Effekt beim Jobangebot zeichnet sich darin nicht ab.

Jedoch ist generell zu beachten, dass die hier berichteten Befunde nicht verallgemeinerbar sind. Zum einen haben Untersuchungen wie die vorliegende einen Fallstudien-Charakter. Zum anderen gibt es im Forschungsfeld insgesamt zu wenige Studien, die den gesamten Bewerberpool einer Organisation (eines Unternehmens) in den Blick nehmen (bisher ausschließlich für die USA). Dennoch sollten auch die Vorteile von Bewerberpoolstudien, die groß angelegte Befragungen von Stellensuchenden und Organisationen ergänzen, deutlich geworden sein. Beispielsweise zeigt unsere Studie, dass nicht auf allen drei Rekrutierungsstufen bestimmte Bewerbergruppen gleichermaßen erfolgreicher sind als andere. Hier deutet sich mit der genauen Verortung von sozialen Prozessen, z.B. mit Blick auf die Vorteile von sozialen Kontakten, ein besonderes Analysepotenzial von Bewerberpoolstudien an.

Die künftige Forschung muss einerseits stärker theoretisch ausgerichtet werden, d.h. auf theoretische Modelle, die sich mit Unterschieden in der Bedeutung von sozialen Kontakten (und Headhuntern) auf einzelnen Rekrutierungsstufen befassen. Eventuell können dazu theoretische Annahmen und Überlegungen in Arbeitsmarktmodellen (z.B. Montgomery 1991) mit Überlegungen zu ,internen“ Organisationskriterien und -abläufen verbunden werden, sodass die ,black box“ Organisation bei der Analyse des Zugangs zu und der Vergabe von Arbeitsplätzen weiter geöffnet wird. Jedoch gilt zu berücksichtigen, dass Ergebnisse von Bewerberpoolstudien mit Blick auf die Vorteile von sozialen Kontakten zum Teil andere Schlussfolgerungen nahe legen als Arbeiten aus der Perspektive von Jobsuchenden (siehe vor allem Mouw 2003 für eine ausführliche, auch theoretisch orientierte, Diskussion). Andererseits sollte, nicht zuletzt deshalb, die Anzahl an Bewerberpoolstudien vergrößert werden. Dabei kann an die wenigen existierenden Untersuchungen angeknüpft werden, die zudem spezielle Bewerbermerkmale in den Blick nehmen (z.B. Petersen et al. 2000 für Geschlecht und ethnische Herkunft der Bewerber), stärker den empfehlenden Mitarbeiter ins Licht rücken (z.B. Yakubovich / Lup 2006) und die Leistungen der erfolgreich rekrutierten Bewerber im Unternehmen untersuchen (z.B. Fernandez et al. 2000). Solche Studien decken neben der Effektivität von Rekrutierungskanälen mitunter ungewollte soziale Prozesse in Organisationen auf (z.B. eine Dis- 
kriminierung von bestimmten Bewerbergruppen) und haben nicht zuletzt deshalb auch eine praktische Relevanz.

\section{Literatur}

Fernandez, Roberto / Nancy Weinberg (1997): Sifting and Sorting: Personal Contacts and Hiring in a Retail Bank, in: American Sociological Review 62, S. 883-902.

Fernandez, Roberto / Lourdes M. Sosa (2005): Gendering the Job: Networks and Recruitment at a Call Center, in: American Journal of Sociology 3, S. 859-904.

Fernandez, Roberto / Emilio J. Castilla / Paul Moore (2000): Social Capital at Work: Networks and Employment at a Phone Center, in: American Journal of Sociology 105, S. 1288-1356.

Fountain, Christine (2005): Finding a Job in the Internet Age, in: Social Forces 83, S. 1235-1262.

Franzen, Axel / Dominik Hangartner (2005): Soziale Netzwerke und beruflicher Erfolg: Eine Analyse des Arbeitsmarkteintritts von Hochschulabsolventen, in: Kölner Zeitschrift für Soziologie und Sozialpsychologie 57, S. 443-465.

Franzen, Axel / Dominik Hangartner (2006): Social Networks and Labour Market Outcomes: The NonMonetary Benefits of Social Capital, in: European Sociological Review 22, S. 353-368.

Frey, Bruno S. (1997): Markt und Motivation: wie ökonomische Anreize die (Arbeits)-Moral verdrängen, München.

Granovetter, Mark [1974] (1995): Getting a Job, 2. Auflage, Chicago.

Holtbrügge, Dirk (2005): Personalmanagement, 2. Auflage, Heidelberg.

Holzer, Harry J. (1987): Job Search by Employed and Unemployed Youth, in: Industrial and Labor Relations Review 40, S. 601-611.

Holzer, Harry J. (1996): What Employers Want? Job Prospects of Less-Educated Workers, New York.

Jann, Ben (2003): Old-Boy Network. Militärdienst und ziviler Berufserfolg in der Schweiz, in: Zeitschrift für Soziologie 32, S. 139-155.

Jung, Hans (2005): Personalwirtschaft, 6. Auflage, München.

Kahn, Lawrence M. / Stuart A. Low (1982): The Relative Effects of Employed and Unemployed Job Search, in: Review of Economics and Statistics 64, S. 234-241.

Marsden, Peter V. / Karen E. Campbell (1990): Recruitment and Selection Processes: The Organizational Side of Job Searches, in: Ronald L. Breiger (Hrsg.), Social Mobility and Social Structure, New York, S. $59-79$.

Marsden, Peter V. / Elizabeth H. Gorman (2001): Social Networks, Job Changes, and Recruitment, in: Ivar Berg / Arne L. Kalleberg (Hrsg.), Sourcebook of Labor Markets, New York, S. 467-502.

Montgomery, James D. (2001): Social Networks and Labor-Market-Outcomes: Toward an Economic Analysis, in: American Economic Review 81, S. 1408-1418.

Mouw, Ted (2003): Social Capital and Finding a Job: Do Contacts Matter?, in: American Sociological Review 68, S. 868-896.

Petersen, Trond / Ishak Saporta / Marc-David L. Seidel (2000): Offering a Job: Meritocracy and Social Networks, in: American Journal of Sociology 106, 763-816.

Preisendörfer, Peter / Thomas Voss (1988): Arbeitsmarkt und soziale Netzwerke: Die Bedeutung sozialer Kontakte beim Zugang zu Arbeitsplätzen, in: Soziale Welt 39, S. 104-119.

Rees, Albert (1966): Information Networks in Labor Markets, in: American Economic Review 56, S. 559-566.

Voss, Thomas (2007): Netzwerke als Sozialkapital im Arbeitsmarkt, in: Axel Franzen/Markus Freitag (Hrsg.), Sozialkapital. Grundlagen und Anwendungen. Sonderheft 47 der Kölner Zeitschrift für Soziologie und Sozialpsychologie, Wiesbaden, S. 321-342. 
Wegener, Bernd (1991): Job Mobility and Social Ties: Social Resources, Prior Job, and Status Attainment, in: American Sociological Review 56, S. 60-71.

Windolf, Paul (1986): Recruitment, Selection, and Internal Labour Markets in Britain and Germany, in: Organization Studies 7 / 3, S. 235-254.

Yakubovich, Valery / Daniela Lup (2006): Stages of the Recruitment Process and the Referrer's Performance Effect, in: Organization Science 6, S. 713-72.

Dr. Ulf Liebe

Georg-August-Universität Göttingen Department für Agrarökonomie und Rurale Entwicklung Lehrstuhl für Soziologie Ländlicher Räume Platz der Göttinger Sieben 5 37073 Göttingen uliebe@uni-goettingen.de

Karsten Wegerich GfK SE

Human Resources

Nordwestring 101

90419 Nürnberg karsten.wegerich@gfk.com 